\title{
Cancer mortality in workers exposed to dieldrin and aldrin: over 50 years of follow up
}

\author{
Ludovic G. P. M. van Amelsvoort • Jos J. M. Slangen • \\ Shan P. Tsai $\cdot$ Geert de Jong $\cdot$ IJmert Kant
}

Received: 6 September 2007 / Accepted: 4 April 2008 / Published online: 22 April 2008

(C) The Author(s) 2008

\begin{abstract}
Objective Dieldrin and aldrin, pesticides widely used until the 1970s, have been under suspicion of being carcinogenic. In this study, overall and cause-specific mortality was assessed in a cohort of 570 employees occupationally exposed to the pesticides dieldrin and aldrin to investigate the long-term health effects, in particular carcinogenic effects.

Methods All of the employees worked in the production plants between January 1954 and January 1970 and were followed for cause-specific mortality until 30 April 2006. Based on dieldrin levels in blood samples taken from 343 workers during the exposure period, the total intake of dieldrin was estimated for each individual subjects in the cohort. The estimated total intake ranged from 11 to $7,755 \mathrm{mg}$ of dieldrin, with an average of $737 \mathrm{mg}$.

Results Two hundred and twenty-six workers had died before 30 April 2006 compared with an expected number of 327.3 , giving a standardized mortality ratio (SMR) of 69.0 (95\% confidence interval (CI): 60.3-78.7). Overall cancer mortality was also significantly lower than expected (SMR: 76.4, 95\% CI: 60.8-94.9). Also, none of the specific cancer sites showed a significant excess mortality and no association between exposure level and cancer mortality was found.
\end{abstract}

L. G. P. M. van Amelsvoort ( $\varangle)$ · J. J. M. Slangen · IJ. Kant Occupational Health Epidemiology, Department of Epidemiology, School for Public Health and Primary Care (Caphri),

Maastricht University, 6200 MD Maastricht, The Netherlands

e-mail: LGPM.vanamelsvoort@epid.unimaas.nl

S. P. Tsai

Shell Health, Shell Oil Company,

Houston, TX, USA

G. de Jong

Shell International, Shell Health,

The Hague, The Netherlands
Conclusion The results from this study support findings from other epidemiological and recent animal studies concluding that dieldrin and aldrin are not likely human carcinogens.

Keywords Aldrin - Carcinogenicity · Dieldrin . Epidemiology $\cdot$ Exposure $\cdot$ Occupation $\cdot$ Cohort

\section{Introduction}

The chlorinated hydrocarbons dieldrin and aldrin were widely used as pesticides in agriculture from the 1950s up to the early 1970s (WHO 1989). Later, their use became more and more restricted to specific applications, such as termite control. They were withdrawn from the market for almost all uses in the USA in 1974 and subsequently in other countries. In 1987, production ceased in the last remaining dieldrin and aldrin producing plant at the Royal Dutch/Shell refinery in Pernis, The Netherlands (de Jong 1991).

Dieldrin and aldrin are readily absorbed following inhalation, ingestion or skin contact. In the occupational setting, the latter is thought to be the most important route of exposure. After uptake, aldrin is rapidly converted to dieldrin, mainly by the P-450 system in the liver. Dieldrin can be stored in adipose tissue

Since 1962, results from animal studies (Davis and Fitzhugh 1962) have spurred concerns that dieldrin and aldrin may be human carcinogens as well. The EPA published a report on the assessment of the human cancer risk of dieldrin and aldrin in 1987 (EPA 1987). In this report, dieldrin and aldrin were classified as probable human carcinogens. This classification was mainly based on evidence of hepatocarcinogenesis in mice. The International Agency for Research on Cancer classified the evidence for 
carcinogenicity in humans as inadequate and animal carcinogenicity as limited (IARC 1987). However, since the EPA assessment of human cancer risk, there is accumulating evidence which has called into question the value of mouse liver tumors as a reliable predictor of carcinogenic potential in humans. Dieldrin-induced oxidative stress or its sequelae apparently result in modulation of gene expression that favors expansion of initiated mouse, but not rat, liver cells; thus, dieldrin acts as a nongenotoxic promoter/accelerator of background liver tumorigenesis in the mouse (Stevenson et al. 1999). Recent animal studies provide a possible explanation for the specific mouse hepatocarcinogenity of aldrin/dieldrin (Stevenson et al. 1999, 1995; Kolaja et al. 1996). More recently, Kamendulis et al. (2001) further unraveled the mechanism and reported that high level dieldrin exposure of mice was accompanied by increased nuclear ploidy and mitosis.

Dieldrin and aldrin were produced at two sites and were formulated in many others. Ditraglia et al. (1981) studied an organochlorine manufacturing plant in Colorado, USA, following 1,155 workers from 1951 to 1977. In the group of dieldrin and aldrin workers, a significant increase in pneumonia and other respiratory diseases was observed. Total cancer mortality was lower than expected. Small and statistically insignificant increases were observed for liver, rectum, esophageal and lymphohaematopoietic neoplasms. The investigators did not regard these findings as effects of the occupational exposures and they recommended further monitoring of the cohort.

In an update of this cohort by Brown, in which the follow-up was extended to 31 December 1987, a statistically significant excess mortality from liver cancer was noted (5 observed deaths vs. 1.3 expected) (Brown 1992). This cohort study was later expanded and updated until 31 December 1990 by Amoateng-Adjepong et al. (1995). The study conducted by Amoateng-Adjepong includes all data collected in the earlier studies on cohorts investigated by Ditraglia and Brown. Therefore, the results of the Amoateng-Adjepong study provide the most complete picture of the mortality experience of the workers of the Colorado plant. Total mortality and all cancer mortality were within the expected range. None of the cause-specific standardized mortality ratios (SMRs) were significantly elevated. During the extended follow-up period between 31 December 1987 and 13 December 1990 no additional deaths from liver cancer were noted.

The second manufacturing plant that has been subjected to extensive epidemiological investigation is the Shell plant at Pernis, near Rotterdam, The Netherlands. Five hundred and seventy workers of this plant, employed between 1954 and 1970, have been followed through 2001 (de Jong et al. 1997; Swaen et al. 2002). The cause-specific mortality patterns of these workers were not different from the expected patterns. A statistically significant increase in rectal cancer was seen: however, it was inversely related to dose. Based on three cases, liver cancer was non-significantly increased in the two lower dose groups, but there were no cases in the highest exposed group (Swaen et al. 2002).

Apart from these two retrospective cohort studies on workers from these production plants, little other epidemiological work has been done on aldrin or dieldrin. Schroeder et al. reported an association between certain subtypes of non-Hodgkin lymphoma and the reported use of dieldrin by farmers (Schroeder et al. 2001). Hoyer et al. (2000), in a Danish study on the survival of breast cancer patients, reported an inverse association between survival and dieldrin serum levels in blood. Recently, Purdue et al. reported a significant positive association between self-reported dieldrin exposure and lung cancer and a significant inverse relation between self-reported aldrin exposure and colon cancer (Purdue et al. 2007). However, the overall results of these three studies seem inconsistent and none of the reported findings have been replicated. For example, a second case/ control study of breast cancer cases and organochlorine traces did not find a relationship between breast cancer and dieldrin concentrations in serum (Ward et al. 2000).

As mentioned earlier, the Pernis plant is one of the few plants that produced dieldrin and aldrin and has the longest record of producing these substances. Therefore the cohort of 570 workers employed at this plant provides a unique opportunity to assess the potential long-term health risk in a population with a high occupational exposure to dieldrin and aldrin. Furthermore, it is the only cohort of its kind where detailed exposure assessment by industrial hygiene data and matching biological monitoring data is available. This exposure assessment was published in detail by de Jong (1991). This study provided data on individual exposures over the years of employment for all subjects who had been employed in the Pernis plants between 1954 (when dieldrin and aldrin production and formulation in this plant began) and 1970. Mortality data from this cohort have been updated and previously assessed by de Jong et al. (1997) and Swaen et al. (2002). With this final update, data are made available with a mean follow-up of 38 years (ranges from 1 to 52 years). Therefore, this update provides a unique opportunity to assess the potential effects on overall and cause-specific mortality from dieldrin and aldrin with an extended latency period.

\section{Methods}

Study population

The population consisted of 570 male employees who worked for at least 1 year in one of the units of the pesticide 
production plants at Pernis between 1 January 1954 and 1 January 1970 . The production plant consisted mainly of an intermediates production plant, an aldrin production plant, a dieldrin production plant and a formulation plant where the final products were mixed and diluted in such a way that they became suitable for agricultural use by customers. Static air sampling in 1958, 1959 and 1960 indicated that the air concentrations in the plant were usually a factor of 5-10 below the threshold limit value as a time weighted average (TLV-TWA) level of $0.25 \mathrm{mg} / \mathrm{m}^{3}$. However, some tasks, such as drum filling, resulted in exposure concentrations as high as $4 \mathrm{mg} / \mathrm{m}^{3}$.

Because of the importance of skin contact to absorption, ambient air measurements are not thought to give an appropriate reflection of exposure. Therefore, estimations of total intake by means of biomonitoring data are regarded as far superior to ambient air monitoring within the given context. An extensive set of biomonitoring data on these workers is available. In the 1960s, several industrial hygiene and biological monitoring programs had been conducted. During the monitoring programs, levels of dieldrin in the blood of workers were determined. Since aldrin is rapidly converted to dieldrin in the body, aldrin levels in blood were not monitored. Between 1963 and 1965, a methanol/hexane extraction method was used. Later, this method was replaced by an acetone/hexane method, which is nearly $100 \%$ accurate. The determination of dieldrin was carried out by gas-liquid chromatography with electron capture detection (de Jong 1991). The biomonitoring was common practice between 1963 and 1970 and varied between every 3 months and once a year.

For 343 of 570 subjects, multiple dieldrin blood measurements are available. From these biomonitoring data, the total intake of dieldrin was calculated using the method described in detail by de Jong (1991). In summary, the association between intake and the resulting blood concentration was studied earlier by means of a human volunteer study in which three groups of volunteers ingested doses of 10 , 50 or $211 \mathrm{mg}$ of dieldrin daily. The relationship between intake and dieldrin in blood was best described by the formula: $C=A-B \mathrm{e}^{-k(t 1-t 0)}$ in which " $C$ " is the dieldrin concentration in blood (in $\mu \mathrm{g} / \mathrm{ml}$ ) attained at time $t 1$ under the assumption of a constant daily intake from time t0. " $A$ " represents the dieldrin concentration in the blood at equilibrium and " $B$ " is the background concentration in the blood originating from other sources, for instance, from food. " $K$ " is the first order rate constant for elimination of dieldrin from the human body. The biological half-life of dieldrin was calculated to be 267 days (Versteeg and Jager 1973). Assuming a stable exposure rate the total intake of dieldrin and aldrin for each worker was estimated based on the biomonitoring data. For the workers with no biomonitoring data, estimates of total intake were made using the biomonitoring data of coworkers with the same job, workplace and time interval.

Total intake of dieldrin and aldrin ranged from 11 to $7,755 \mathrm{mg}$, accumulated during their work at the plants up to 1970. In 1970, several major improvements were made in the working environment and processes, and exposure to dieldrin and aldrin was greatly reduced. The effects of these improvements have been demonstrated by decreases in dieldrin levels in blood. Using the individual total dieldrin and aldrin intake estimates, the population was stratified into three groups (with 190 workers in each group): low, moderate and high levels of total intake. The arithmetic mean of total intake in the low group $(n=190)$ was $270 \mathrm{mg}$ of dieldrin and aldrin. In the moderate intake group $(n=190)$ the mean was $540 \mathrm{mg}$, and in the high group $(n=190)$ it was $750 \mathrm{mg}$. Alongside the stratification of the exposed workers into the three subgroups, we conducted analyses for the specific jobs in the plants. We identified four different jobs, namely assistant operator $(n=165)$, maintenance worker $(n=83)$, operator $(n=302)$ and supervisor $(n=20)$. It was estimated that the highest exposures occurred in assistant operators and operators of the formulation plant and in the assistant operators of the dieldrin and aldrin production plants.

\section{Follow-up}

The total cohort was followed for mortality until 30 April 2006. By means of the Dutch Municipal Population Registries, information was collected on the vital status of each study subject. For deceased workers, the underlying cause of death was obtained from the Central Bureau of Statistics.

Ascertainment of vital status and causes of death

The procedures that were applied to obtain the vital status and the causes of death were similar to the previous study. The municipal population registries (about 460 in The Netherlands in 2006) were requested to provide information on the whereabouts of the workers that were included in this study. For workers who had moved from one municipality to another, the new municipality was requested to provide vital status information on that particular worker. This process was repeated after each notification that a person had moved. In this way, all of the 570 ex-workers were traced.

Another route for identification of vital status was by consulting a special registry for persons who had left The Netherlands by means of emigration. It was noted that quite a lot of people who had emigrated during some time in their lives returned to The Netherlands after retirement. Checking the data provided by this registry revealed additional information on former workers. As a result, these persons 
were no longer considered lost to follow-up and their person years were calculated and added to the total person years of follow-up. (More detailed information on vital status is shown in Table 1.)

In the last step in identifying the individual causes of death for all the deceased former employers death certificate data was retrieved from the Central Bureau of Statistics (CBS). The CBS receives a copy of all Dutch death certificates after a person's death. After the receipt of the death certificates, the causes of death are coded by trained nosologists and computerized to accumulate the annual vital statistics, which are presented by causes of death. For all deceased workers, the cause of death was identified in this database.

\section{Statistics}

The observed cause-specific mortality of the cohort was compared with the expected number based on age and time interval cause-specific mortality rates of the total male Dutch population. SMRs were calculated by dividing the observed numbers of deaths by the expected numbers $(\times 100)$, and appropriate $95 \%$ confidence limits (CI) were calculated with an exact method based on the Poisson distribution. This was done using the SAS Macro as described by Daly (1992). Since employees were classified into three groups of cumulative exposure (total intake), the expected numbers of deaths were calculated based on the personyears of observation accumulated after termination of exposure as described by Swaen and Volovics (1987).

\section{Results}

As of 30 April 2006, 226 workers had died, adding 55 deaths to the earlier follow-up (see Table 1). Nine persons were lost to follow up, as they were not registered by the communal personal administration any more. The total number of person-years of observation time was 21,702. The 226 total observed deaths were significantly lower than the expected number of 327.3, resulting in a SMR of 69.0 (95\% CI: 60.3-78.7). A detailed overview of total and cause specific mortality can be found in Table 2 . For all four categories of major causes of death, the number of observed deaths was lower than the expected and none of the cause-specific SMRs was significantly elevated. In fact, nearly all cause-specific SMRs were below 100. The SMR for liver and biliary passages cancer, the cancer of primary interest based on the mouse data, was 216.1 (95\% CI: 58.9553.9) based on four observed cases.

To assess a potential relationship with cumulative exposure, an exposure level stratified analysis was performed (Table 2) using three groups with 190 workers per group. The low-intake group had a cumulative intake between 11 and $201 \mathrm{mg}$ of aldrin and/or dieldrin. The intake of the moderate group ranged from 203 to $732 \mathrm{mg}$. Workers in the high-intake group all had estimated intakes ranging from 737 to $7,755 \mathrm{mg}$, with an arithmetic mean of $1,704 \mathrm{mg}$. In all the three dose groups, the mortality for all causes was significantly lower than the general population of The Netherlands with SMRs of 75.1, (95\% CI: 57.2-96.9), 72.1 (95\% CI: 57.0-90.0), and 67.0 (95\% CI: 53.8-82.4) for the low, moderate and high dose groups, respectively. When looking at the overall mortality due to neoplasms, all SMRs were the same or below 100 with a downward trend with increasing cumulative exposure. For the high-intake group, the mortality for neoplasms was significantly lower than the Dutch general population $(\mathrm{SMR}=66.2,95 \% \mathrm{CI}: 44.0$ 95.6). With respect to liver and skin malignancies, there were non-statistical excesses in the total group $(\mathrm{SMR}=216.1,95 \%$ CI: 58.9-553.9 and $\mathrm{SMR}=302.4$, 95\% CI: $62.4-883.8$, respectively), but no deaths were observed in the high-intake group. For rectal cancer, a nonstatistical excess in the total group was observed $(\mathrm{SMR}=214.8,95 \%$ CI: 78.8-467.6), a small and non-significant excess mortality in the high-intake group was also observed (SMR $=175.6,95 \%$ CI: 21.3-634.3), but no clear trend with exposure was observed. Similar pattern of no trend with exposure was seen for oesophagus cancer. The overall mortality risk for bladder cancer was decreased
Table 1 Vital status ascertainment on 1 May 2006 for 570 workers exposed the dieldrin and aldrin between 1 January 1954 and 1 January 1970

\begin{tabular}{|c|c|c|c|c|c|c|}
\hline \multirow[t]{2}{*}{$\begin{array}{l}\text { Vital status at end } \\
\text { date of follow-up }\end{array}$} & \multicolumn{2}{|c|}{$\begin{array}{l}\text { Follow-up } \\
\text { until } 1 \text { January } 1993\end{array}$} & \multicolumn{2}{|c|}{$\begin{array}{l}\text { Follow-up } \\
\text { until } 1 \text { January } 2001\end{array}$} & \multicolumn{2}{|c|}{$\begin{array}{l}\text { Follow-up } \\
\text { until } 1 \text { May } 2006\end{array}$} \\
\hline & $N$ & $(\%)$ & $N$ & $(\%)$ & $N$ & $(\%)$ \\
\hline Alive & 402 & 70.5 & 335 & 58.8 & 297 & 52.1 \\
\hline Emigrated & 35 & 6.2 & 47 & 8.2 & 38 & 6.7 \\
\hline Lost to follow-up & 15 & 2.6 & 17 & 3.0 & 9 & 1.6 \\
\hline Deceased & 118 & 20.7 & 171 & 30.0 & 226 & 39.6 \\
\hline $\begin{array}{l}\text { Number of person- } \\
\text { years at risk }\end{array}$ & $16,297.28$ & & $19,704.56$ & & $21,702.0$ & \\
\hline Total group & 570 & 100 & 570 & 100 & 570 & 100 \\
\hline
\end{tabular}




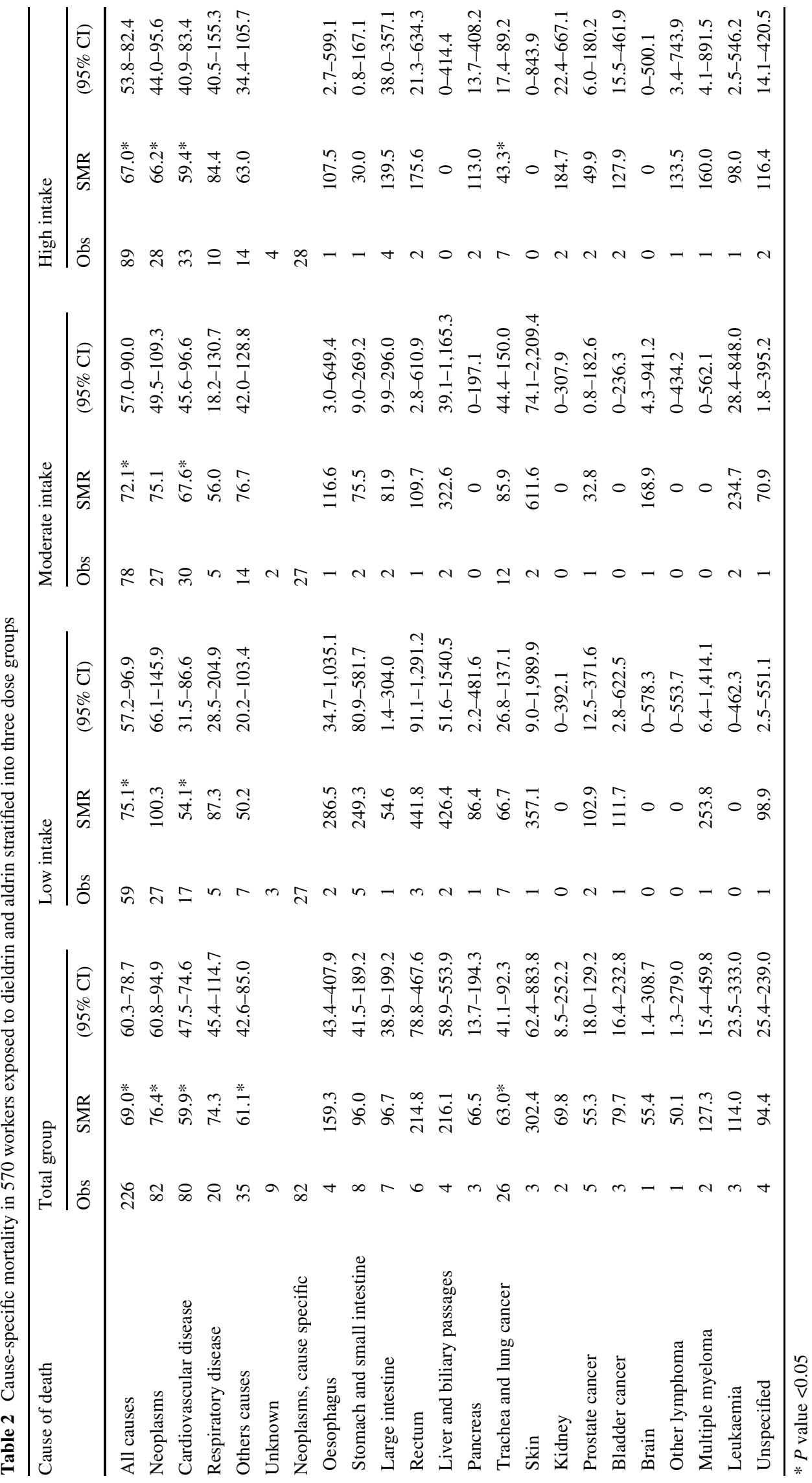


$(\mathrm{SMR}=79.7,95 \%$ CI: 16.4-232.8) although it was slightly elevated, albeit non-significant, in the highest intake group $(\mathrm{SMR}=127.9,95 \%$ CI: $15.5-461.9)$.

The sub classification by job held (Table 3 ) revealed a significantly lowered mortality from lung cancer (SMR: 43.4, 95\% CI: 19.8-82.3) and significantly elevated number of skin cancers (SMR: $575.8,95 \%$ CI: $118.8-1,682.8$ ) in the operators group.

\section{Discussion}

After 52 years of follow up, this cohort of 570 workers exposed to dieldrin and aldrin does not demonstrate any excess cancer mortality risk that could be related to exposure. For overall and most specific cancer types, the observed numbers of deaths were lower than expected based on the cause-specific mortality rates of the total male Dutch population. The only significantly elevated SMR (skin cancer in operators) is most likely a chance finding that can be attributed to the number of statistical comparisons performed in this study. There were no additional cases since the previous update (Swaen et al. 2002). None of the other epidemiological or animal studies available report a similar finding.

When considering potential exposure to dieldrin and aldrin, mortality from liver cancer is of interest based on mouse studies. In two updates of a study first conducted by Ditraglia et al. (1981), the investigators found an increase in deaths from cancer of the liver and biliary passages (de Jong et al. 1997; Swaen et al. 2002). The current study found an increase, although statistically insignificant, of deaths from cancer of the liver and biliary passages but no increasing dose-response relationship. There were four deaths, two in the lowest exposure group and two in the moderate exposure group, but none of the deaths occurred in the highest exposure group. So this breakdown by intake does not indicate statistically significantly increased SMRs and does not suggest an increasing cancer risk with increasing dose. Of the two deaths in the moderate exposure group, one was primary liver carcinoma and the other was from cancer of the gall bladder. The individual with liver cancer worked at Pernis for about 2 years after having worked as a fisherman and sailor for the previous 40 years. This individual had a medical history suggestive of a nonoccupational risk factor for liver cancer. These results make a causal association of liver and biliary passages cancer with aldrin or dieldrin unlikely.

It is to be noted that the observed number of deaths from cancer of the rectum was statistically greater than expected in the previous two studies of this cohort, although none showed a dose-response relation. Between 1993 and 2006, there was no new rectal cancer death, and the mortality risk (i.e., SMR) has been decreased from 390 (95\% CI: 140-850) in the original study (de Jong et al. 1997) to 300 (95\% CI: 109-649) in the 2001 update study (Swaen et al. 2002), and to 216 (95\% CI: 59-554) in the current study. In addition, no deaths were observed in the high intake group.

This cohort of workers provides us with one of the few possibilities to evaluate the long-term health effects of relatively high dieldrin/aldrin exposure levels in a human population. Moreover, this study also incorporated data on estimated intake of dieldrin for individual cohort members, based on blood samples from 343 workers during the period in which exposure had occurred. Cumulative intake of the 570 study subjects varied between 11 and $7,755 \mathrm{mg}$, with an average of $737 \mathrm{mg}$. It is estimated that over $75 \%$ of the cohort had dieldrin exposure levels that exceeded the assumed human equivalent dose rate corresponding to the lowest positive dose rate for female mice in a cancer bioassay in which the incidence of liver tumors had doubled.

Sielken et al. (1999), based on an earlier study of this cohort, have reported a cancer risk assessment for dieldrin and aldrin. The overall mortality for cancer of that study was slightly lower than the Dutch general population (46 observed deaths with an SMR of 96.8, 95\% CI =71-129). When examining cancer risks by levels of exposure, the SMRs were 118.9 (95\% CI=63.2-203.3), 102.1 (95\% $\mathrm{CI}=58.3-165.8)$ and $81.4(95 \% \mathrm{CI}=47.4-130.3)$ for the low, moderate and high exposure groups, respectively. Based on lifetime average daily dose in $\mu \mathrm{g} / \mathrm{kg}$ body weight/ day of dieldrin and aldrin, the study found that there were not an increase in cancer risks of $10^{-6}$ at lifetime average dose of 0.0000625 or $10^{-4}$ at 0.00625 as would be estimated using US Environmental Protection Agency's upper bound on cancer potency based on mouse liver tumors. In fact, there was no observed increase in cancer risk in these workers at doses as large as $2 \mu \mathrm{g} /(\mathrm{kg}$ day $)$. Since the mortality pattern of the current study is the same as that reported earlier, results of cancer potency assessment from the previous analysis would apply to this study, probably with a narrower confidence interval.

It is not likely that bias with respect to ascertainment and coding of the causes of death may have affected the study outcome. Information regarding the causes of death was collected from the CBS where the causes of death were coded at the time of death by trained nosologists who were unaware of our study and were unaware of which persons were or were not a member of the cohort. Equally, information regarding exposure, including the calculation of total intake, was performed without any knowledge of the vital status and cause of death if applicable. Also, the number of subjects lost to follow-up in this study is low when considering the long period of follow up. This follow up has even been able to trace some of the respondents, which were lost-to-follow up in previous updates, due to remigration and improvements in the registries. 


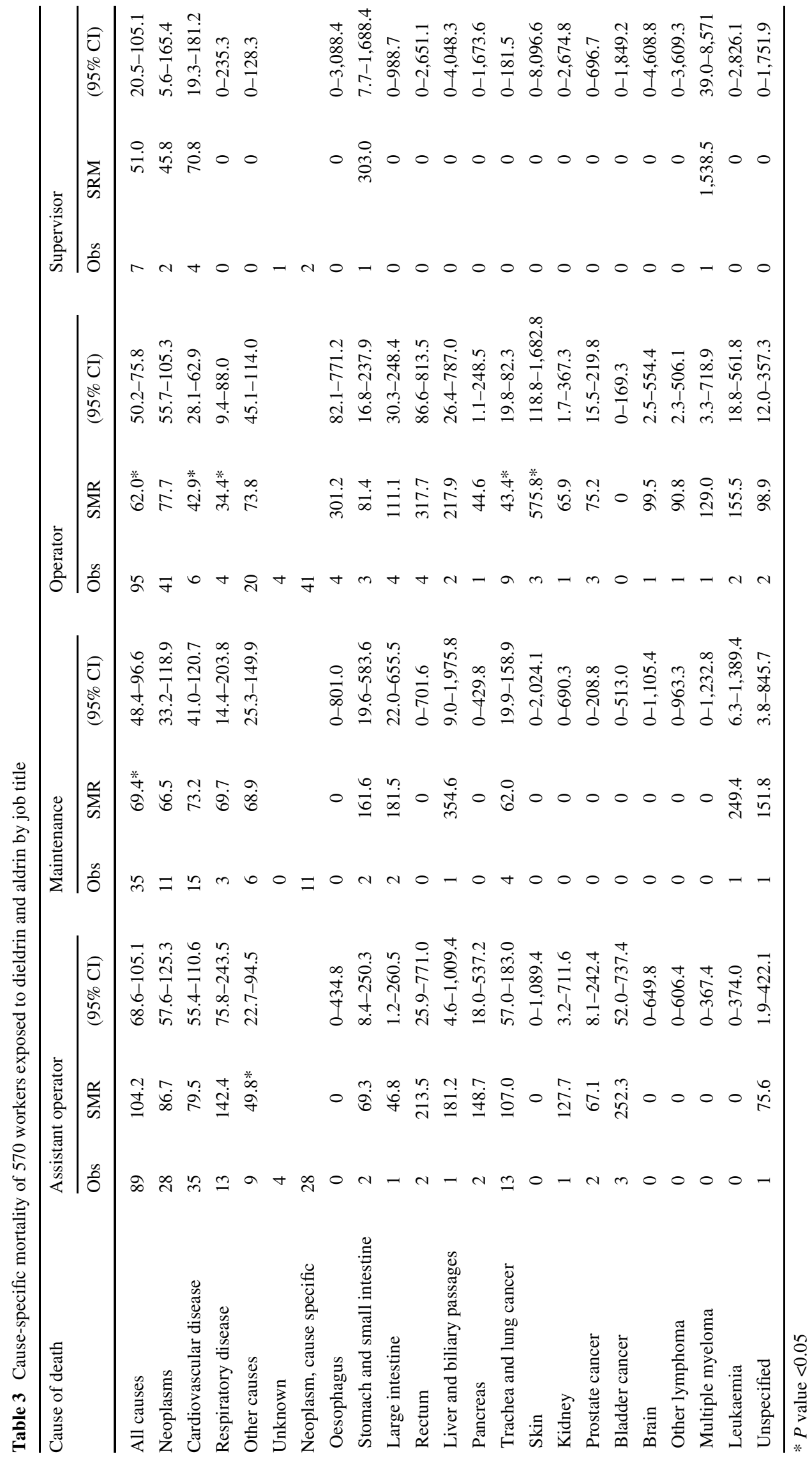


A limitation of the study is its relatively small sample size. However, the power of a retrospective cohort study depends on the number of expected events of interest, in this case cancer deaths, in combination with the expected magnitude of the effect of the exposure. In fact, given an $\alpha$ level of 0.05 and $80 \%$ power, the sample size (i.e. person years) of this study is capable of detecting at least a $34 \%$ increase risk in cancer (Armstrong 1987), if such a risk did exist. However, as none of the cancers revealed a significant excess mortality risk and no exposure response relationship was observed for any of the cancer sites, this follow up study supports the conclusion that aldrin/dieldrin exposure does not lead to an increased cancer risk in man.

This cohort is one of two cohorts that have been involved in the production of dieldrin and aldrin in the world. Their exposure has been accurately documented and as such provides an excellent opportunity to learn more about the possible long-term effects of these pesticides. In addition, the time window of observation is 52 years, between 1 January 1954 and 30 April 2006, which is a sufficient latency period. In fact, all exposed workers were employed before 1 January 1970 and $52.3 \%$ before 1 January 1960. Our findings add to the growing body of evidence, provided by both epidemiological studies (Amoateng-Adjepong et al. 1995; Ward et al. 2000) and recent animal studies (Stevenson et al. 1999; Kamendulis et al. 2001), showing a lack of an association between aldrin/dieldrin exposure and cancer mortality.

The overall mortality of this occupational cohort remains significantly lower than the general male population of the Netherlands, after 52 years of follow-up. This is commonly referred to as the healthy worker effect (Checkoway et al. 1989), which can be attributed to a number of factors ( $\mathrm{Li}$ and Sung 1999). However, it is somewhat unexpected to find that the overall SMR decreased compared to the previous update while the majority of the cohort has reached their retirement age. One explanation is that the cohort members of this present study are healthier. The lack of complete ascertainment of death is also a possible reason, however, it is not likely since the lost to follow-up was extremely low, only $1.6 \%$. Furthermore, as $70-80 \%$ of the reference population is also working, the finding of such a decreased risk is less likely to be totally explained by the healthy worker effect. A similar observation has been reported by others; the SMR was 74.7 in the original study (Enterline et al. 1990) but decreased to 60.7 in an additional 10-year follow-up (Tsai et al. 1996).

A longer follow-up would provide more precise risk estimates and a better understanding of the relationship between exposures and disease. However, a recent study has suggested that increasing follow-up could decrease the risk estimate of occupational cohorts (Silver et al. 2002). Some also postulated that risk estimates could be "diluted" with increasing follow-up if the exposure acts as a promoter rather than an initiator (Lamm et al. 1989). Nevertheless, the potential negative impact of extending follow-up has not been well understood and requires further studies.

In conclusion, our study supports the results of other extensive epidemiological studies of workers exposed to dieldrin and aldrin. That is that there is no evidence of an increased mortality risk for cancer of any particular type as a result of exposure to aldrin or dieldrin.

Acknowledgments This study was supported by Shell International.

Open Access This article is distributed under the terms of the Creative Commons Attribution Noncommercial License which permits any noncommercial use, distribution, and reproduction in any medium, provided the original author(s) and source are credited.

\section{References}

Amoateng-Adjepong Y, Sathiakumar N, Delzell E, Cole P (1995) Mortality among workers at a pesticide manufacturing plant. J Occup Environ Med 37(4):471-478

Armstrong B (1987) A simple estimator of minimum detectable relative risk, sample size, or power in cohort studies. Am J Epidemiol 126(2):356-358

Brown DP (1992) Mortality of workers employed at organochlorine pesticide manufacturing plants-an update. Scand J Work Environ Health 118(3):155-161

Checkoway H, Pearce N, Crawford-Brown D (1989) Research methods in occupational epidemiology. Oxford University Press, New York

Daly L (1992) Simple SAS macros for the calculation of exact binomial and Poisson confidence limits. Comput Biol Med 22(5):351-361

Davis KJ, Fitzhugh OG (1962) Tumorigenic potential of aldrin and dieldrin for mice. Toxicol Appl Pharmacol 4:187-189

Ditraglia D, Brown DP, Namekata T, Iverson N (1981) Mortality study of workers employed at organochlorine pesticide manufacturing plants. Scand J Work Environ Health 7(Suppl 4):140-146

Enterline PE, Henderson V, Marsh G (1990) Mortality of workers potentially exposed to epichlorohydrin. Br J Ind Med 47(41):269-276

EPA (Environmental Protection Agency) (1987) Carcinogenicity assessment of aldrin and dieldrin. EPA, Washington DC

Hoyer AP, Jorgensen T, Grandjean P (2000) Breast cancer and dieldrin. Lancet 356(9244):1852-1853

IARC (1987) Evaluation of carcinogenic risk of chemicals to humans. Overall evaluations of carcinogenicity: an update of IARC monographs, vols 1-42. IARC (International Agency for Research on Cancer, Lyon

de Jong G (1991) Long-term health effects of aldrin and dieldrin. A study of exposure, health effects and mortality of workers engaged in the manufacture and formulation of the insecticides aldrin and dieldrin. Toxicol Lett Suppl:1-206

de Jong G, Swaen GM, Slangen JJ (1997) Mortality of workers exposed to dieldrin and aldrin: a retrospective cohort study. Occup Environ Med 54(10):702-707

Kamendulis LM, Kolaja KL, Stevenson DE, Walborg EF Jr., Klaunig JE (2001) Comparative effects of dieldrin on hepatic ploidy, cell proliferation, and apoptosis in rodent liver. J Toxicol Environ Health A 62(2):127-141

Kolaja KL, Stevenson DE, Johnson JT, Walborg EF Jr., Klaunig JE (1996) Subchronic effects of dieldrin and phenobarbital on hepatic DNA synthesis in mice and rats. Fundam Appl Toxicol 29(2):219-228 
Lamm SH, Walters AS, Wilson R, Byrd DM, Grunwald H (1989) Consistencies and inconsistencies underlying the quantitative assessment of leukemia risk from benzene exposure. Environ Health Perspect 82:289-297

Li CY, Sung FC (1999) A review of the healthy worker effect in occupational epidemiology. Occup Med (Lond) 49(4):225-229

Purdue MP, Hoppin JA, Blair A, Dosemeci M, Alavanja MC (2007) Occupational exposure to organochlorine insecticides and cancer incidence in the agricultural health study. Int $\mathrm{J}$ Cancer 120(3):642-649

Schroeder JC, Olshan AF, Baric R, Dent GA, Weinberg CR, Yount B et al (2001) Agricultural risk factors for $t(14 ; 18)$ subtypes of nonHodgkin's lymphoma. Epidemiology 12(6):701-709

Sielken RL Jr., Bretzlaff RS, Valdez-Flores C, Stevenson DE, de Jong $\mathrm{G}$ (1999) Cancer dose-response modeling of epidemiological data on worker exposures to aldrin and dieldrin. Risk Anal 19(6):1101-1111

Silver SR, Rinsky RA, Cooper SP, Hornung RW, Lai D (2002) Effect of follow-up time on risk estimates: a longitudinal examination of the relative risks of leukemia and multiple myeloma in a rubber hydrochloride cohort. Am J Ind Med 42(6):481-489

Stevenson DE, Walborg EF Jr., Klaunig JE (1995) The species specificity of dieldrin- or phenobarbital-induced hepatocarcinogenesis: case studies with implications for human health risk assessment. Prog Clin Biol Res 391:337-345

Stevenson DE, Walborg EF Jr, North DW, Sielken RL Jr, Ross CE, Wright AS et al (1999) Monograph: reassessment of human cancer risk of aldrin/dieldrin. Toxicol Lett 109(3):123-186

Swaen GM, Volovics A (1987) Investigating dose response relations in occupational mortality studies: something to keep in mind. $\mathrm{Br}$ J Ind Med 44(9):642-644

Swaen GM, de Jong G, Slangen JJ, van Amelsvoort LG (2002) Cancer mortality in workers exposed to dieldrin and aldrin: an update. Toxicol Ind Health 18(2):63-70

Tsai SP, Gilstrap EL, Ross CE (1996) Mortality study of employees with potential exposure to epichlorohydrin: a 10 year update. Occup Environ Med 53(5):299-304

Versteeg JP, Jager KW (1973) Long-term occupational exposure to the insecticides aldrin dieldrin, endrin, and telodrin. $\mathrm{Br} \mathrm{J}$ Ind Med 30(2):201-202

Ward EM, Schulte P, Grajewski B, Andersen A, Patterson DG Jr., Turner W et al (2000) Serum organochlorine levels and breast cancer: a nested case-control study of Norwegian women. Cancer Epidemiol Biomarkers Prev 9(12):1357-1367

WHO (World Health Organization) (1989) Aldrin and dieldrin. Environ Health Criteria 91:1-335 